Jens Klose

\title{
Negativzinsen, Überschussreserven und Tiering der EZB: Wie stark sind die Banken belastet?
}

\begin{abstract}
Die Europäische Zentralbank hat im Juni 2014 ihren Einlagezinssatz erstmals in den negativen Bereich gesenkt. Seitdem ist er in mehreren Stufen bis auf heute $-0,5 \%$ gesunken. Banken haben dadurch zunehmend Probleme, ihre Einlagen ohne großes Risiko und nominalen Wertverlust anzulegen. Der negative Einlagezins war aber noch nie die effektive Untergrenze für Einlagen bei der EZB und durch die Entscheidung im September 2019 ist, insbesondere durch das neu eingeführte Tiering-Programm der EZB, der Durchschnittszins sogar gestiegen und nicht etwa gefallen. Durch dieses Programm hat die EZB für Entlastung gesorgt, von der die Banken im Euroraum aber unterschiedlich profitieren.
\end{abstract}

\begin{abstract}
Die Europäische Zentralbank ist für die Geldpolitik im Euroraum zuständig. Diese Aufgabe erfüllt sie hauptsächlich über das Setzen eines Leitzinses. Seit Ausbruch der Finanzkrise 2008/2009 sind zwar weitere Instrumente hinzugekommen, die hier allerdings nur indirekt eine Rolle spielen. Neben dem Leitzins, genauer gesagt dem Hauptrefinanzierungssatz, setzt die EZB noch zwei weitere Zinssätze, die einen Zinskorridor aufspannen. Dies sind als obere Grenze der Satz der Spitzenrefinanzierungsfazilität und als untere Grenze der Satz der Einlagefazilität, kurz der Einlagezins. Zu letzterem können Banken bei der EZB überschüssiges Kapital über Nacht einlegen.
\end{abstract}

Die EZB hat, wie viele Zentralbanken in entwickelten Volkswirtschaften, den Leitzins nach der Finanzkrise rapide gesenkt, sodass dieser momentan bei $0 \%$ liegt. Das hat fast zwangsläufig zur Folge, dass der Einlagezins negativ sein muss. Seit Juni 2014 ist dies tatsächlich der Fall. Bis heute ist der Einlagezins damit der einzige

(C) Der/die Autor(en) 2020. Open Access: Dieser Artikel wird unter der Creative Commons Namensnennung 4.0 International Lizenz (https:// creativecommons.org/licenses/by/4.0/deed.de) veröffentlicht.

Open Access wird durch die ZBW - Leibniz-Informationszentrum Wirtschaft gefördert.

Prof. Dr. Jens Klose ist Professor für Statistik und allgemeine Volkswirtschaftslehre an der Technischen Hochschule Mittelhessen in Gießen.
Zins, den die EZB in den negativen Bereich gesetzt hat. Gleichwohl hat diese Maßnahme, gerade in Deutschland, ein großes Echo nach sich gezogen. Häufig sind in deutschen Medien oder von deutschen Politikern Begriffe wie „Strafzinsen“ oder Redewendungen wie die „Enteignung des deutschen Sparers" zu hören. Diesen Vorwürfen hat sich jüngst die deutsche Vertreterin im EZB-Direktorium, Isabel Schnabel, entgegengestellt (Schnabel, 2020).

Durchschnittliche Verzinsung von

Überschussliquidität bei der EZB

Die EZB operiert seit Beginn der Finanzkrise mal mehr mal weniger in einem System mit Überschussliquidität, also mit Einlagen, die über die Mindestreserve hinausgehen. Diese Überschussliquidität ist in erster Linie durch die Politiken der EZB auf der Aktivseite ihrer Bilanz begründet. Hierzu zählen insbesondere diverse Refinanzierungsgeschäfte und Ankaufprogramme der EZB, welche die Bilanzsumme erheblich ausgeweitet haben. Diese Politiken zeigen sich demnach spiegelbildlich auf der Passivseite der EZB-Bilanz. Während individuelle Kreditinstitute z.B. durch Kreditvergabe ihre Überschussliquidität senken können, ist dies im gesamten Bankensystem nur schwer möglich. Lediglich durch Bargeldhaltung wäre dies zu erreichen. Da diese aber mit Kosten verbunden ist, mag sich eine Einlage bei der EZB trotz negativer Zinsen als lohnender erweisen (Klose, 2013). So ist bis heute nicht zu erkennen, dass sich der Anstieg der Bargeldhaltung im Euroraum signifikant geändert hat. Die aus negativen Einlagezinsen resultierenden Kosten sind bis heute offenbar geringer als die Kosten der eigenen Bargeld-Lagerung.

Wenn das überschüssige Geld also wieder bei der EZB angelegt wird, welche Möglichkeiten bleiben den Kredit- 
instituten dies zu tun? Bei der EZB sind hierfür zwei Konten vorgesehen: zum einen die bereits erwähnten Konten der Einlagefazilität und zum anderen die Girokonten, die in normalen Zeiten für die Verwendung der Mindestreserve genutzt werden. Es ist aber auch möglich, mehr Geld als die Mindestreserveverpflichtung, der sogenannte Mindestreserve-Soll, hier einzulegen. Während die Mindestreserve, deren geldpolitische Bedeutung es ist, eine exzessive Kreditvergabe zu verhindern, indem ein bestimmter Prozentsatz jeder Einlage bei einer Bank bei der EZB hinterlegt werden muss, grundsätzlich mit dem Leitzins verzinst wird, werden darüber hinausgehende Einlagen auf den Girokonten in der Regel nicht verzinst, weshalb in normalen Zeiten für jede Bank ein Anreiz besteht, überschüssige Liquidität in der Einlagefazilität anzulegen, um wenigsten einen gewissen Zinsertrag zu erwirtschaften. Der durchschnittliche Zinssatz, den eine Bank auf die Einlagen bei der EZB erhält, ist somit:

$$
i_{\varnothing}=\frac{i_{L Z} \star M R+i_{G K} * \ddot{U} G+i_{E F} * E F}{M R+\ddot{U} G+E F}
$$

Hier ist $i_{\varnothing}$ der Durchschnittszins einer Bank, $i_{L Z}$ der Leitzins, $i_{G K}$ der Zins für Einlagen auf Girokonten, die über die Mindestreservepflicht hinausgehen, $i_{E F}$ der Einlagezins und $M R$ die Mindestreserveverpflichtung, ÜG die Überschüsse auf den Girokonten, die über die Mindestreserve hinausgehen, sowie EF die Einlagen in der Einlagefazilität. Die Summe aus ÜG und EF wird dabei typischerweise als Überschussliquidität bezeichnet.

Ein Beispiel soll die drei Möglichkeiten einer Einlage bei der EZB verdeutlichen: Der Leitzins soll $2 \%$ betragen und der Einlagezins 1,5\%. Eine Bank muss eine Mindestreserve von 1 Mio. Euro halten, legt insgesamt 8 Mio. Euro in den Girokonten und nochmal 8 Mio. Euro in der Einlagefazilität an. Somit erhält man einen Durchschnittszins:

$$
i_{\varnothing}=\frac{2 \% * 1+0 \% * 7+1,5 \% * 8}{1+7+8}=0,875 \%
$$

Seit mehr als fünf Jahren ist der Einlagezins nun aber bereits negativ. Mehr noch: Mit der Einführung des negativen Einlagezinses hat die EZB auch den Zinssatz für die über die Mindestreserveanforderung hinausgehenden Einlagen auf den Girokonten von null auf den negativen Einlagezins umgestellt, um keine Ausweichreaktionen zu schaffen. Zudem ist der Leitzins momentan bei genau $0 \%$. Dadurch dreht sich ebenfalls der Durchschnittszins einer Einlage bei der EZB in den negativen Bereich, sobald nur 1 Euro Überschussliquidität eingelegt wird.

Das Beispiel kann man auf diese veränderten Rahmenbedingungen anpassen, indem man die Zinssätze verändert:
Der Leitzins beträgt nun $0 \%$ und der Einlagezins, sowie der Zins für die Überschussreserven auf den Girokonten jeweils $-0,5 \%$. Dadurch ergibt sich ein Durchschnittszins:

$$
i_{\varnothing}=\frac{0 \% * 1+(-0,5 \%) * 7+(-0,5 \%) * 8}{1+7+8} \approx-0,469 \%
$$

Aus diesem Beispiel wird sofort deutlich, dass der durchschnittliche Zins auf Einlagen bei der EZB stets höher als der negative Einlagezins ist, solange die Mindestreserve einen positiven Wert annimmt, was solange der Fall ist, wie Banken Einlagen der Kunden erhalten und die EZB einen positiven Mindestreservesatz erhebt.

In der Sitzung im September 2019 hat der EZB-Rat neben der Absenkung des Einlagezinses von -0,4\% auf -0,5\% die Einführung eines sogenannten Tiering-Systems beschlossen, also eines zweistufigen Systems zur Verzinsung der Überschussreserven. Dieses System trat erstmals in der Mindestreserve-Erfüllungsperiode vom 30. Oktober bis 17. Dezember 2019 in Kraft. Das Ziel des Tiering-Systems ist es, „die bankbasierte Transmission der Geldpolitik zu unterstützen und sicherzustellen, dass die negativen Zinssätze auch weiterhin einen positiven Beitrag zum akkommodierenden geldpolitischen Kurs leisten“ (Europäische Zentralbank, 2019). Tatsächlich rückt mit einer höheren Überschussliquidität der kurzfristige Interbankenzins näher an den Einlagezins und schwankt eben nicht länger um den Leitzins, wie in normalen Zeiten. Die EZB hat erkannt, dass das momentane Niveau der Überschussliquidität weit über dem Niveau liegt, das nötig ist, um den Interbankenzins auf dem Niveau des Einlagezinses zu fixieren. Deshalb wurde das Tiering-System eingeführt, mit dem ein Teil der Überschussliquidität vom negativen Zinssatz ausgenommen wird und so die Durchschnittsverzinsung der Banken bei der EZB automatisch angehoben wird. Dabei muss das Ausmaß der Freistellung so bestimmt werden, dass es dadurch zu keinen signifikanten Zinsanstiegen kommt, welche die Wirkung negativer Einlagezinsen konterkarieren würden.

Die EZB setzt dieses System so um: Der Betrag, der von den Negativzinsen ausgenommen ist und stattdessen nicht verzinst wird, bemisst sich als ein Vielfaches des Mindestreserve-Solls eines jeden Kreditinstituts. Dieses Vielfache oder der Multiplikator ist dabei für jedes Kreditinstitut identisch. Um den Freibetrag nutzen zu können, muss die Überschussliquidität auf den Girokonten liegen. Ein Abzug bei der Einlagefazilität ist nicht möglich. Rein technisch ist dies aber keine große Hürde, da das Geld relativ einfach von einem Konto bei der EZB ins andere gebucht werden kann. Demnach verändert sich die obige Formel der Durchschnittsverzinsung durch das TieringSystem wie folgt: 


$$
i_{\varnothing}=\frac{i_{L Z^{*}} M R+0 \% * T M * M R+i_{G K} *(\ddot{G}-T M * M R)+i_{E F} * E F}{M R+\ddot{U} G+E F}
$$

Es kommt hierdurch also eine mit $0 \%$ verzinste Komponente hinzu, die sich als Tiering-Multiplikator (TM) multipliziert mit der Mindestreserve bemisst. Diese Überschussliquidität ist bei den sonstigen Überschüssen in den Girokonten abzuziehen. Die EZB hat den Tiering-Multiplikator auf 6 gesetzt, der aber jederzeit verändert werden kann. Demnach ergäbe sich im Beispiel ein Durchschnittszins von:

$i_{\varnothing}=\frac{0 \% * 1+0 \% * 6 * 1+(-0,5 \%) *(7-6 * 1)+(-0,5 \%) * 8}{1+7+8} \approx-0,281 \%$

Allein durch die Einführung des Tierings hat sich die Durchschnittsverzinsung also um rund 0,2 Prozentpunkte erhöht. Die Gleichung und das Beispiel zeigen aber auch deutlich, dass die optimale Ausnutzung des Tiering-Systems nur gelingt, wenn die Banken genügend Überschussliquidität in den Girokonten gelagert haben. Es kann zu Fällen kommen, wo dies nicht gegeben ist. Dies wäre so, wenn im Beispiel weniger als 6 Mio. Euro Überschussliquidität auf den Girokonten gehalten werden würden. Im Einzelfall mag dies über eine entsprechende Umschichtung der Überschussliquidität weg von der Einlagefazilität hin zu den Girokonten kompensiert werden können. Falls auch dies nicht möglich ist, würde ein Teil der zinsbefreiten Überschussliquidität ungenutzt bleiben. In den folgenden Berechnungen wird stets von einer optimalen Ausnutzung des Tiering-Systems durch die Banken ausgegangen. Die ersten Auswertungen der EZB und der Bundesbank stützen diese Annahme, da es durch entsprechende Umschichtungen mit rund 95\% zu einer fast vollständigen Ausnutzung der Freibeträge in der ersten Erfüllungsperiode kam, die in der zweiten sogar leicht anstieg (Europäische Zentralbank, 2019; Deutsche Bundesbank, 2020).

Überschussliquidität im Euroraum und deren Komponenten

Wie hat sich die Überschussliquidität im Euroraum in den letzten Jahren entwickelt? Abbildung 1 zeigt die Einlagen bei der EZB gemäß den beschriebenen vier Komponenten. Die Abbildung beginnt Mitte 2008, da mit dem Kollaps der Investmentbank Lehman Brothers in den USA im September 2008 zum ersten Mal ein signifikantes Maß an Überschussliquidität, also Einlagen die über die Mindestreserve hinausgehen, zu verzeichnen war.

Die überschüssige Liquidität wurde zunächst ausschließlich in der Einlagefazilität angelegt, was sinnvoll ist, da es zu diesem Zeitpunkt hierfür eine positive Verzinsung war, wohingegen die Überschussreserven in den Girokonten unverzinst geblieben wären. Den ersten größeren Auf-

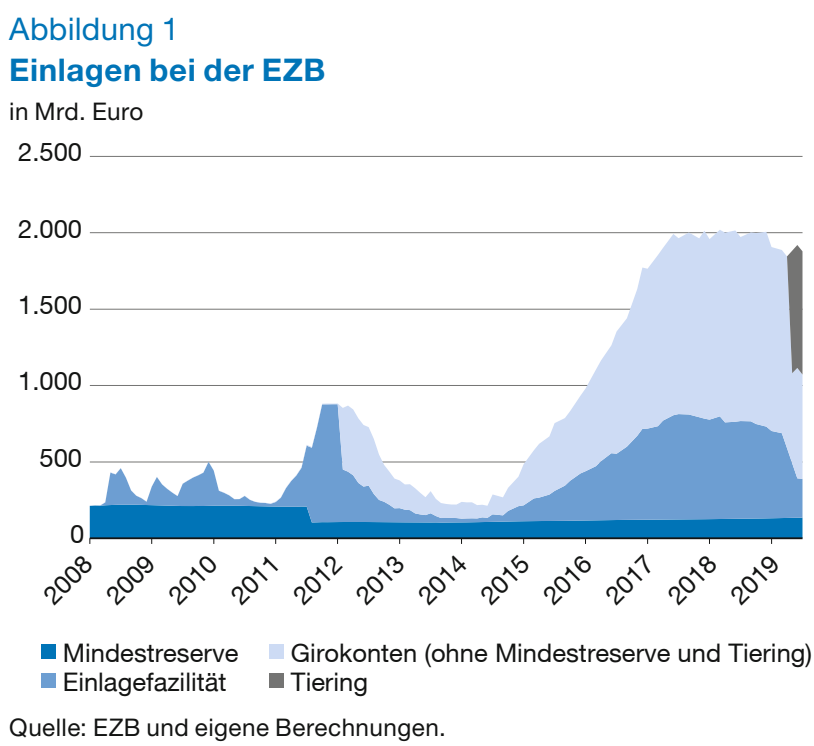

wuchs erfährt die Überschussliquidität Ende 2011 bis Anfang 2012. Dies hängt unmittelbar mit den beiden zu dieser Zeit begebenen Refinanzierungsgeschäften mit dreijähriger Laufzeit zusammen, die zusammen ein Volumen von über 1 Billion Euro aufwiesen. In diesen Zeitraum fällt zudem die Senkung des Mindestreservesatzes von $2 \%$ auf $1 \%$ (Europäische Zentralbank, 2011). Mitte 2012 wird dann zum ersten Mal ein signifikanter Anteil der Überschussliquidität auf den Girokonten angelegt. Ursache ist, dass ab Juli 2012 der Einlagesatz auf $0 \%$ gesenkt wurde und die Banken damit de facto indifferent waren, ob sie ihre Überschussliquidität auf den Girokonten oder der Einlagefazilität anlegen sollten (Sachverständigenrat zur Begutachtung der gesamtwirtschaftlichen Entwicklung, 2012). Ab diesem Zeitpunkt sind die Verzinsung für Einlagefazilität und Überschussreserven in den Girokonten stets identisch, weshalb es aus Sicht der Banken egal ist, auf welchem der beiden Konten die Gelder deponiert werden.

Der zweite bis heute andauernde Anstieg beginnt Anfang 2015, da die EZB zu diesem Zeitpunkt ein Aufkaufprogramm eingeführt hat, das vor allem Staatsanleihen, aber auch Unternehmensanleihen, Asset Backed Securities und Pfandbriefe umfasst. In dieser Zeit ergibt sich auch das Maximum der Einlagen bei der EZB mit rund 2 Billionen Euro. Die Überschussliquidität ist dabei zum einen in der Einlagefazilität, zu einem größeren Teil aber in den Girokonten eingelegt. Das Verhältnis von Überschussliquidität zu Mindestreserve betrug Mitte 2018 beispielweise rund 14,7. Die Auswirkungen des Tiering-Systems werden ganz zum Ende des Untersuchungszeitraums ersichtlich. Insbesondere kam es durch die Einführung zu einer Verschiebung in der Einlagestruktur weg von der Nutzung der Einlagefazilität, hin zur Anlage in den Girokonten. Dies ist verständlich, da nur letztere Einlagen auch für die Nutzung des Tierings infrage kommen. 


\section{Abbildung 2}

Einlagen bei der Bundesbank

in Mrd. Euro

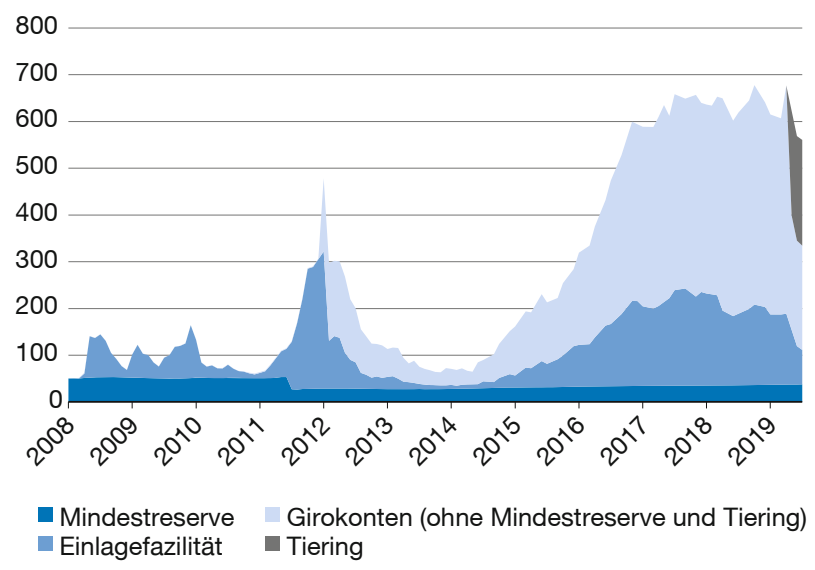

Quelle: Deutsche Bundesbank und eigene Berechnungen.

Einen ähnlichen Verlauf wie für das Eurosystem als Ganzem sieht man auch bei den nationalen Zentralbanken. Exemplarisch wird dies hier für die Deutsche Bundesbank (vgl. Abbildung 2) und die Banca d'Italia (vgl. Abbildung 3) dargestellt. Während bei der Bundesbank allerdings stets überproportional viele Überschussreserven anfallen, ist bei der Banca d'Italia genau das Gegenteil der Fall. Als Beispiel sei wieder Mitte 2018, also der Hochpunkt der Einlagen bei der EZB gewählt. Hier beträgt das Verhältnis der Überschussliquidität zu der zu haltenden Mindestreserve bei der Deutschen Bundesbank rund 17,1 und damit um 2,4 mehr als für das Eurosystem als Ganzem, während es bei der Banca d'Italia nur bei 3,8 liegt. Dies ist eine direkte Folge der grenzüberschreitenden Überweisungen von Banken, die sich in den Target2-Salden ausdrücken. ${ }^{1}$ Tatsächlich sieht man insbesondere im Jahr 2012 und ab 2015 eine rapide Zunahme der Target2-Überschüsse der Bundesbank und spiegelbildlich eine Zunahme der Target2-Defizite der Banca d'Italia.

Man sieht ebenfalls, dass sich das Tiering-System deutlich auf die Überschussliquidität bei beiden nationalen Zentralbanken ausgewirkt hat. In beiden Fällen sinkt die Überschussliquidität, die weiterhin mit einem Negativzins belegt ist, dramatisch, was genau das Ziel dieser Maßnahme war. Um besser zu verstehen, welche Anpassungen sich durch die Einführung des Systems ergeben haben, zeigt Tabelle 1 eine Gegenüberstellung zwischen der letzten Periode ohne Tiering-System, die am 29. Oktober 2019 endete, und der ersten Periode

1 Vgl. für eine Diskussion der Target2-Salden z. B.: Sinn und Wollmershäuser (2011)
Abbildung 3

Einlagen bei der Banca d'Italia

in Mrd. Euro

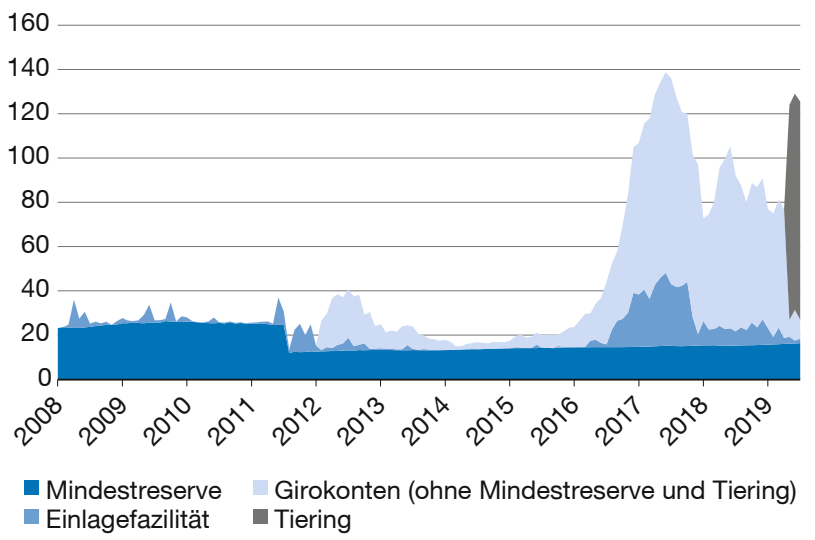

Quelle: Banca d'Italia und eigene Berechnungen.

mit Tiering-System, die am 17. Dezember 2019 zu Ende ging. Es zeigen sich substanzielle Veränderungen durch die Einführung des Tiering-Systems: So nimmt die dem Negativzins unterliegende Überschussliquidität wie zu erwarten dramatisch ab. Im Vergleich zum Oktober unterliegen im Dezember nur noch rund $57 \%$ dem negativen Einlagezins. Über 800 Mrd. Euro werden, statt mit einem Negativzins belegt zu werden, nicht mehr verzinst. Durch das Tiering steigt auch der Durchschnittszins an. Betrug er noch im Oktober knapp über $-0,5 \%^{2}$, lag er im Dezember rund 21 Basispunkte höher. Damit ist durch die Entscheidungen der EZB im September 2019, als neben der Einführung des Tiering-Programms auch eine Absenkung des Einlagezinses von $-0,4 \%$ auf $-0,5 \%$ beschlossen wurde, de facto ein spürbarer Anstieg des Durchschnittszinses zu verzeichnen. Dies schlägt sich dann auch in einer entsprechenden Zinsersparnis nieder. So sparten die Banken gegenüber dem Referenzszenario vor der Septemberentscheidung, also mit einem Einlagezins von $-0,4 \%$, fast 280 Mio. Euro in der Erfüllungsperiode. Wenn es lediglich die Senkung des Einlagezinses ohne die Einführung des Tierings gegeben hätte, wäre die Belastung für die Banken sogar um über 500 Mio. Euro höher gewesen.

Um den gesamten Effekt der negativen Einlagezinsen zu umgehen, hätte der Multiplikator des Tierings bei rund 13 liegen müssen. Er steigt durch die Zunahme der Überschussliquidität im Dezember auch nur leicht an. Allerdings ist zu beachten, dass eine vollständige

2 Dass der Durchschnittszins knapp darüber liegt, wird schlicht von der kleinen Menge Mindestreserve verursacht, welche die Banken bei der EZB halten mussten und die nicht verzinst wurde. 
Tabelle 1

Auswirkungen des Tiering-Systems im Euroraum in Deutschland und in Italien

\begin{tabular}{|c|c|c|c|c|c|c|c|c|c|}
\hline & \multicolumn{3}{|c|}{ Euroraum } & \multicolumn{3}{|c|}{ Deutschland } & \multicolumn{3}{|c|}{ Italien } \\
\hline & $\begin{array}{c}\text { Oktober } \\
2019\end{array}$ & $\begin{array}{c}\text { Dezember } \\
2019\end{array}$ & $\begin{array}{l}\text { Ände- } \\
\text { rung }\end{array}$ & $\begin{array}{c}\text { Oktober } \\
2019\end{array}$ & $\begin{array}{c}\text { Dezember } \\
2019\end{array}$ & $\begin{array}{l}\text { Ände- } \\
\text { rung }\end{array}$ & $\begin{array}{c}\text { Oktober } \\
2019\end{array}$ & $\begin{array}{c}\text { Dezember } \\
2019\end{array}$ & $\begin{array}{l}\text { Ände- } \\
\text { rung }\end{array}$ \\
\hline Überschussliquidität (in Mrd. Euro) & 1711,87 & 981,13 & $-730,74$ & 639,72 & 307,89 & $-331,83$ & 60,61 & 15,16 & $-45,45$ \\
\hline Tiering (in Mrd. Euro) & - & 804,81 & 804,81 & - & 225,66 & 225,66 & - & 97,66 & 97,66 \\
\hline Durchschnittszins in \% & $-0,464$ & $-0,251$ & 0,213 & $-0,472$ & $-0,29$ & 0,182 & $-0,395$ & $-0,058$ & 0,337 \\
\hline Multiplikator & 12,85 & 13,31 & 0,46 & 17,13 & 14,26 & $-2,87$ & 3,76 & 6,93 & 3,17 \\
\hline Zinsersparnis gegenüber -0,4 \% (in Mio. Euro) & - & 279,76 & 279,76 & - & 73,35 & 73,35 & - & 46,93 & 46,93 \\
\hline Zinsersparnis gegenüber $-0,5$ (in Mio. Euro) & - & 503,01 & 503,01 & - & 139,8 & 139,8 & - & 61,04 & 61,04 \\
\hline
\end{tabular}

Durchschnittszins nach den Formeln im Text, Multiplikator nötig, um negative Einlagezinsen komplett zu umgehen.

Quelle: eigene Berechnungen.

Abschöpfung der Überschussliquidität keinen Sinn ergeben würde, da damit mittelbar auch der kurzfristige Geldmarktzins ansteigen würde und der negative Einlagezins somit obsolet wäre. Die Ergebnisse scheinen insgesamt robust zu sein, da sich für die im Januar 2020 endende Erfüllungsperiode ein ähnliches Bild wie im Dezember ergibt.

Die Einführung des Tiering-Systems hat allerdings unterschiedliche Auswirkungen auf die Banken in den einzelnen Staaten. Exemplarisch soll dies an einem Vergleich von Deutschland und Italien gezeigt werden. In beiden Staaten geht die mit dem negativen Einlagezins belegte Überschussliquidität zurück. Allerdings sinkt sie in Italien mit rund $75 \%$ wesentlich stärker als in Deutschland mit lediglich ca. $52 \%$. Zählt man das Geld, das dem Tiering unterliegt, hinzu, so fällt auf, dass die gesamte Überschussliquidität in Deutschland um rund 106 Mrd. Euro zurückgeht, in Italien hingegen um ca. $52 \mathrm{Mrd}$. Euro steigt. Es kommt durch das Tiering-Programm also zu einer Reallokation der Liquidität im Bankensektor des Euroraums.

Dies hat insbesondere dazu geführt, dass sich die in Deutschland viel beachteten Target2-Salden wieder angenähert haben. So sind die Target2-Forderungen der Bundesbank von Oktober 2019 bis Januar 2020 um insgesamt rund $64 \mathrm{Mrd}$. Euro und die Target2-Verbindlichkeiten der Banca d'Italia im gleichen Zeitraum um ungefähr 70 Mrd. Euro zurückgegangen. Das Tiering-Programm sollte also insbesondere auch denjenigen gefallen, die in den Target 2-Salden erhebliche Verlustrisiken für die nationalen Zentralbanken der Überschussländer sehen.
Doch warum kommt es zu der Reallokation von Liquidität im Euroraum? Der Grund liegt einfach in der optimalen Ausnutzung des Tiering-Vorteils. Lag im Oktober in Deutschland der Multiplikator zur kompletten Überführung der Überschussliquidität in das Tiering-System bei über 17, betrug er in Italien weniger als 4 . Dementsprechend wären in Italien ohne Reallokation alle Überschussreserven unter das Tiering-System gefallen und es wäre darüber hinaus ein signifikanter Tiering-Betrag ungenutzt geblieben. Durch die Reallokation sinkt der Multiplikator im Dezember in Deutschland auf nur noch rund 14 und steigt in Italien auf fast 7 und damit über den von der EZB gesetzten Wert von 6.

Diese unterschiedlichen Multiplikatoren, haben auch substanzielle Unterschiede in der Durchschnittsverzinsung zur Folge. Zwar profitieren die Banken beider Länder von der Maßnahme insofern, als in beiden Ländern die Durchschnittsverzinsung steigt, dieser Effekt ist aber in Italien wesentlich größer als in Deutschland. Dies ist logisch, da in Italien aktuell fast die gesamte Überschussliquidität über das Tiering-System mit 0 verzinst wird und nur ein sehr geringer Teil dem negativen Einlagezins unterworfen ist. In Deutschland wird hingegen immer noch mehr als die Hälfte der Überschussliquidität mit dem negativen Einlagezins verzinst. Trotz dieser Unterschiede sparen die Banken beider Länder durch das Tiering-System Zinsen. Dies gilt unabhängig davon, ob ein Negativzins von - $0,4 \%$ oder $-0,5 \%$ unterstellt wird.

\section{Fazit}

Negativzinsen in einem Umfeld, das durch Überschussliquidität gekennzeichnet ist, stellen Banken vor Herausfor- 
derungen. Es entstehen Zinsverluste. Diese Verluste aus der Einlage bei der EZB fallen aber niemals in voller Höhe des Negativzinses an. Vielmehr ist ein Durchschnittszins zu bilden, der zum einen die Mindestreservepflicht und zum anderen das neu geschaffene Tiering-System miteinbezieht. Besonders mit letzterem hat die EZB ein Instrument geschaffen, das es ihr bei richtigem Einsatz erlaubt, die akkomodierende Geldpolitik über negative Zinsen beizubehalten, ohne die Banken über Gebühr zu belasten. Hierfür muss lediglich der Multiplikator möglichst groß gewählt werden, unter der Einschränkung, dass es zu keinem signifikanten Anstieg der kurzfristigen Geldmarktzinsen kommt. Momentan scheint dies gut zu gelingen. Allerdings ist auch der Durchschnittszins trotz Tiering noch immer im negativen Bereich. Sollte die EZB dies ändern wollen, bestünde die Möglichkeit, die Mindestreserve wie auch die Liquidität im Tiering-System mit einem positiven Zinssatz zu verzinsen. Allerdings ist hier zu beachten, dass dies für die EZB Verluste induzieren würde, da sie Liquidität momentan zum Nullzins vergibt. Ein Kompromiss könnte so aussehen, dass man den Zins für Mindestreserve und Tiering so austariert, dass dadurch der Durchschnittszins $0 \%$ beträgt.

\section{Literatur}

Deutsche Bundesbank (2020), Deutsche Bundesbank Monatsbericht, Februar 2020, 23-24, Frankfurt a. M.

Europäische Zentralbank (2011, 8. Dezember), ECB announces measures to supportbank lending and money market activity, Pressemitteilung, Frankfurt a. M.

Europäische Zentralbank (2019), EZB-Wirtschaftsbericht, Ausgabe 8/2019, 51 ff., Frankfurt a. M.

Klose, J. (2013), Negative Einlagezinsen im Euroraum? Lehren aus Dänemark, Wirtschaftsdienst, 93(12), 824-827, https://www.wirtschaftsdienst.eu/inhalt/jahr/2013/heft/12/beitrag/negative-einlagezinsenim-euroraum.html (1. April 2020).

Sachverständigenrat zur Begutachtung der gesamtwirtschaftlichen Entwicklung (2012), Jahresgutachten 2012/13: Stabile Architektur für Europa - Handlungsbedarf im Inland, Ziffer 137, Wiesbaden.

Schnabel, I. (2020, 11. Februar), Narrative über die Geldpolitik der EZB - Wirklichkeit oder Fiktion?, Rede bei der juristischen Studiengesellschaft, Karlsruhe.

Sinn und Wollmershäuser (2011, 24. Juni), Target-Kredite, Leistungsbilanzsalden und Kapitalverkehr: Der Rettungsschirm der EZB, ifo Schnelldienst, Sonderausgabe.

Title: Negative interest rates, excess reserves and tiering of the ECB: How heavily are banks burdened?

Abstract: The European Central Bank (ECB) reduced its deposit rate into negative territory in June 2014. It has been reduced further in several steps to reach today's level of $-0.5 \%$. Banks are increasingly facing problems investing their deposits without taking great risks and avoiding nominal depreciation. This article shows, however, that the deposit rate has never been the effective lower bound for deposits at the ECB and, moreover, the decision in September 2019 to introduce the tiering programme increased the average deposit rate rather than decreased it as one would have supposed. With this programme, the ECB has relaxed the situation, even though banks in the euro area make different gains.

JEL Classification: E52, E58 